\title{
Cytokines, Angiogenesis, and Extracellular Matrix Degradation are Augmented by Oxidative Stress in Endometriosis
}

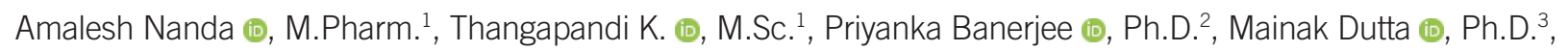

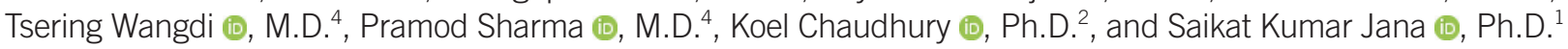

${ }^{1}$ Department of Biotechnology, National Institute of Technology, Yupia, Papum Pare, Arunachal Pradesh, India; ${ }^{2}$ School of Medical Science and Technology, Indian Institute of Technology Kharagpur, West Bengal, India; ${ }^{3}$ Department of Biotechnology, Birla Institute of Technology and Science, Pilani, Dubai Campus, Dubai International Academic City, Dubai, UAE; “ Department of Obstetrics and Gynecology, Pratiksha Hospital, Borbari, Guwahati, Assam, India

Background: The effect of the interplay among inflammation, angiogenesis, extracellular matrix (ECM) degradation, and oxidative stress (OS) on the pathogenesis of endometriosis remains unclear. Previously, we demonstrated the role of OS in endometriosis. Here, we performed a comprehensive investigation of several molecules involved in inflammation, angiogenesis, and ECM degradation in women with endometriosis to study their interplay with OS.

Methods: Blood samples were collected from women with endometriosis $(\mathrm{N}=80)$, as well as from women with tubal factor infertility as controls $(\mathrm{N}=80)$. Interleukin (IL)-1 $1 \beta$, tumor necrosis factor-alpha, interferon-gamma, transforming growth factor-beta, IL-4, -10, -2, -6, -8 , vascular endothelial growth factor (VEGF), matrix metalloproteinase (MMP)-2, -9, tissue inhibitor of metalloproteinases (TIMP)-1, -2, and cyclooxygenase (COX)-2 levels in serum samples were measured using an ELISA. Nuclear factor kappa-light-chain-enhancer of activated $\mathrm{B}$ cells $(\mathrm{NF}-\mathrm{kB})$ in peripheral blood mononuclear cells was measured using flow cytometry.

Results: Cytokines, VEGF, MMPs, and COX-2 were significantly higher and TIMPs were significantly lower in patients with endometriosis. Multivariate statistical analysis indicated that IL-10 was the most significant variable capable of discriminating endometriosis samples from controls.

Conclusions: Deregulation of NF-kB activation by OS affects the expression of various cytokines in endometriosis. Elevated cytokine levels further up-regulate IL-10, which subsequently activates the MMPs, leading to excessive ECM degradation and angiogenesis. Moreover, IL-10 emerged as the most important molecule involved in the pathogenesis of endometriosis. Measurement of these molecules may help in better management of the patients with endometriosis.

Key Words: Oxidative stress (OS), Endometriosis, Extracellular matrix, Cytokines
Received: September 17, 2019

Revision received: January 26, 2020

Accepted: April 1, 2020

\section{Corresponding author:}

Saikat Kumar Jana, Ph.D.

Department of Biotechnology, National Institute of Technology, Yupia, District:

Papum Pare, Arunachal Pradesh-791112, India

Tel: +91-9485230608

Fax: +91-360-2284972

E-mail: saikatmicro4@gmail.com

\section{(c) (i) (5)}

(๔) Korean Society for Laboratory Medicine

This is an Open Access article distributed under the terms of the Creative Commons Attribution Non-Commercial License (https://creativecommons.org/licenses/by-nc/4.0) which permits unrestricted non-commercial use, distribution, and reproduction in any medium, provided the original work is properly cited.

\section{INTRODUCTION}

Endometriosis is a gynecological disorder affecting nearly $10 \%$ of the female population during their reproductive age [1]. It is characterized by the growth of endometrial stroma and glands at extra-uterine sites. It is commonly associated with localized inflammation in the pelvis [2] and increased growth of endometriotic lesions in the presence of oxidative stress (OS) [3]. OS is 
characterized by increased intracellular levels of reactive oxygen species (ROS). ROS provoke the up-regulation of inflammatory cytokine molecules through distinct molecular mechanisms. They regulate activation of nuclear factor kappa-light-chain-enhancer of activated $\mathrm{B}$ cells (NF-kB), which is associated with immune and inflammatory responses [4]. The link between $\mathrm{NF}-\mathrm{\kappa B}$ activation and inflammation in endometriosis has been demonstrated [5, 6]. In recent years, the possible role of ROS in regulating the mediators of inflammation [7], angiogenesis [8] and matrix degradation [9] has received increasing attention.

Cytokines, known as important mediators of intercellular communication, are also produced by the endometriotic implants. Cytokines appear to play vital roles in the development and progression of endometriosis and associated infertility [5]. Aberrant expression of cytokines may also induce extracellular matrix (ECM) degradation [8, 9]. Matrix metalloproteinases (MMPs) are reported to play a pivotal role in establishing ectopic endometrial growth in endometriosis. The endometrial expression of MMPs is regulated by different growth factors, steroid hormones, and cytokines [12]. For endometrial tissue implantation and growth to occur in ectopic sites, the tissue must first attach itself to the host tissue, then invade it by obtaining its own blood supply from the local vasculature [13]. This implantation of ectopic endometrial fragments is based on neovascularization to guarantee a supply of oxygen and essential nutrients [14]. Vascular endothelial growth factor (VEGF) has emerged as a chief regulator of normal angiogenesis and pathological neovascularization in endometriosis [15-17].

There is overwhelming evidence supporting that inflammation, angiogenesis, ECM degradation, and OS play the key roles in the pathogenesis and progression of endometriosis [15, 18]. However, the effect of the interplay among these factors on disease pathogenesis remains unclear. Previously, we demonstrated the role of OS in endometriosis [19]. The present study is an extension of our previous work, in which we showed that several OS markers, including catalase, lipid peroxidation, superoxide dismutase, total antioxidant capacity, glutathione, and advanced oxidation protein products, are dysregulated in patients with endometriosis. Thus, we performed a comprehensive investigation of the molecules involved in inflammation, angiogenesis, and ECM degradation in patients with endometriosis to study their role in disease pathogenesis. In addition, we identified the most important molecule involved in endometriosis pathogenesis. Finally, we propose a model explaining the interplay among various molecules in the disease.

\section{MATERIALS AND METHODS}

\section{Patient selection and sample collection}

This nested case-control retrospective study was performed from August 2016 to July 2018. The necessary ethical approval was obtained from the Institutional Scientific and Ethical Committee of National Institute of Technology, Arunachal Pradesh, India [Proposal\# NIT(A.P)/R\&D/Project/11-12/1/Nol.2/IEC-02] prior to commencing this study. Written informed consent was obtained from the patients prior to blood collection. Blood samples $(\mathrm{N}=80)$ were collected at Pratiksha Hospital, Guwahati, Assam, India, from women with endometriosis, confirmed by diagnostic laparoscopy followed by a biopsy. Samples $(\mathrm{N}=80)$ were also collected from women with tubal factor infertility who served as controls. Tubal factor infertility corresponds to women undergoing salpingectomy for ectopic pregnancy or proximal tubal obstruction due to low-grade infection or fimbrial occlusion with or without mild peritubular adhesions. The women included in the study did not receive any medical or hormonal treatment during the last three months. Moreover, women with a prior history of any gynecological surgery, including chocolate cyst, lower pelvic and abdominal surgery, with other possible causes of pain or pelvic pathology, including pelvic tuberculosis, were excluded from the study. Venous blood samples $(10 \mathrm{~mL})$ were collected in sterile vacutainers by medical practitioners during the daytime from patients in fasting state and in their early follicular phase, as described previously [19, 20]. The collected blood samples were allowed to clot and centrifuged at 2,000 $\times \mathrm{g}$ for 10 minutes at $4^{\circ} \mathrm{C}$. The supernatant serum samples were transferred to sterile tubes and stored at $-80^{\circ} \mathrm{C}$ till further analysis of various cytokines, angiogenic factors, matrix-degrading proteases and their inhibitors. Anthropometric parameters such as age, body mass index (BMI), and estrogen levels were obtained from the patient data sheet. Serum estrogen level was measured using the Estradiol E2 Human ELISA Kit (Abcam, Cambridge, UK).

\section{Measurement of various cytokines, angiogenic molecules, and MMPs}

The levels of interleukin (IL)-1 $\beta$, tumor necrosis factor-alpha (TNF- $\alpha$ ), interferon-gamma (IFN- $\gamma$ ), transforming growth factorbeta (TGF- $\beta 1$ ), IL-4, -10, -2, -6, -8, VEGF, MMP-2, -9, tissue inhibitor of metalloproteinase (TIMP)-1, -2, and cyclooxygenase-2 (COX-2) were evaluated using ELISA, as described previously [7]. Briefly, human IL-1 $\beta$, TNF- $\alpha$, IFN- $\gamma$, and IL-10, -2, -4, -6, and -8 levels were measured using the corresponding ELISA 
kits (R\&D Systems, Minnesota, USA), whereas TGF- $\beta 1$, MMP-2, -9, TIMP-1, -2, and COX-2 levels were determined using an anti-human Rb polyclonal antibody against TGF- $\beta 1$, MMP-2, -9, TIMP-1, -2, and COX-2 (Santa Cruz Biotechnology, Inc., Santa Cruz, CA, USA), respectively. VEGF was determined using an anti-human Rb polyclonal antibody against VEGF (Santa Cruz Biotechnology, Inc.), employing a quantitative indirect enzyme immunoassay technique. All assays and calibrations were performed in triplicate. In cases of very high or low results, the measurements were repeated. The intra- and inter-assay variability were, respectively: $2.5 \%$ and $3.11 \%$ for IL-1 $\beta, 2.7 \%$ and $5.3 \%$ for TNF- $\alpha, 3.2 \%$ and $4.3 \%$ for IFN- $\gamma,<7 \%$ and $<8 \%$ for TGF- $\beta 1,3.7 \%$ and $2.6 \%$ for IL-4, $2.1 \%$ and $5.8 \%$ for IL-10, $3.1 \%$ and $3.3 \%$ for IL-2, $4.1 \%$ and $7.9 \%$ for IL-6, $4 \%$ and $3.2 \%$ for IL-8, 5.3\% and $7.6 \%$ for VEGF, $6 \%$ and $<7 \%$ for MMP-2, $<5 \%$ and $<7 \%$ for MMP-9, $<5 \%$ and $<8 \%$ for TIMP- 1 , $<5 \%$ and $<6 \%$ for TIMP-2, and $<8 \%$ and $<12 \%$ for COX-2.

\section{Measurement of NF- $\kappa B$ in peripheral blood mononuclear cells (PBMCs)}

To establish the role of NF-kB in the pathogenesis of endometriosis, its expression in PBMCs was investigated. After immediate collection of venous blood samples, PBMCs were isolated as reported previously [21]. Next, $10 \mathrm{~mL}$ of peripheral whole blood was loaded with $3.8 \%$ sodium citrate $(9: 1 ; \mathrm{v} / \mathrm{v})$ onto a FicollPaque Plus gradient (GE17-1440-02, Sigma, India). The mix was then centrifuged for 30 minutes at $500 \times g$ at room temperature. The band containing PBMCs was separated, and the cells were washed three times in phosphate-buffered saline (PBS) solution, $\mathrm{pH}$ 7.4. The cells were then examined under a microscope and counted independently by two investigators. The number of the cells was fixed to $1 \times 10^{6} \mathrm{PBMCs} / \mathrm{mL}$. Measurement of NF-kB in PBMCs was performed as described by Ichiyama, et al. [22]. Briefly, the cells were labeled with human specific mouse NF-kB antibodies (nuclear-localized signal p-50 and p-65; sc-8414 and sc-8008, Santa Cruz Biotechnology, Inc.). Cells were then tagged with fluorescein isothiocyanate-conjugated rat anti-mouse lgG3 monoclonal antibody (Pharmingen, San Diego, CA, USA). After washing, the cells were fixed with $1 \%$ paraformaldehyde in PBS and subjected to flow cytometry. Mean fluorescence data acquisition was performed in Cell Quest Pro software attached to a fluorescence activated cell sorter (FACS Calibre, M/S Becton, Dickinson and Company, Franklin Lakes, New Jersey, USA). All experiments were performed in triplicate.

\section{Statistical analysis}

Data analysis using t-tests was performed with Key Plot version 2.0 beta 13 (https://kyplot.software.informer.com/2.0/) and GraphPad QuickCalcs, (GraphPad Software Inc., San Diego, CA, USA) following data normalization. Statistical significance was defined as $P \leq 0.05$. All data are presented as mean \pm SD. Multivariate statistical analysis, including principal-component analysis (PCA) and partial least square discriminate analysis (PLS-DA), was performed using SIMCA 15.0.2 (Umetrics, Malmö, Sweden). PCA, an unsupervised modeling approach, was used to identify a general trend in the samples. Supervised modeling, PLS-DA, was used to identify the most relevant variable responsible for the separation of endometriosis samples from controls. The quality of the PLS-DA model was determined using R2 (goodness of fit) and Q2 (goodness of prediction) values. Furthermore, the model was cross-validated by permutation test statistics using 100 different permuted models.

\section{RESULTS}

The demographic data (Table 1) did not show any significant difference in age and BMI between women with endometriosis and the controls. Serum estrogen levels were significantly higher in patients with endometriosis. Table 2 summarizes the expression levels of various cytokines. All cytokine levels were found to be significantly higher in women with endometriosis than in controls. The level of VEGF was also significantly higher in patients with endometriosis (Table 2). While MMP-2, -9 and COX-2 levels were significantly higher, TIMP-1 and -2 levels were significantly lower in women with endometriosis than in controls (Table 2).

As shown in Fig. 1, NF-kB ( $p-50$ and $p-65$ ) expression was increased in patients with endometriosis than in controls. Finally, multivariate statistical analysis was used to identify the most significant variable responsible for discrimination of endometriosis cases from the control group (Fig. 2). The scores scatter plot (Fig. 2A) generated using PCA analysis showed that the variables can be used to distinctively classify the two groups in an unbiased manner. The PLS-DA scores scatter plot indicated

Table 1. Demographics of the patients recruited for the study

\begin{tabular}{lccc}
\hline Parameters & Endometriosis* $^{*}$ & Control $^{*}$ & $P$ \\
\hline Age $(\mathrm{yr})$ & $32.08 \pm 6.09$ & $30.43 \pm 4.39$ & 0.827 \\
BMI $\left(\mathrm{kg} / \mathrm{m}^{2}\right)$ & $28.24 \pm 0.78$ & $27.37 \pm 0.60$ & 0.379 \\
Serum estrogen level $(\mathrm{ng} / \mathrm{L})$ & $228.10 \pm 12.08$ & $181.60 \pm 11.45$ & 0.007 \\
\hline
\end{tabular}

* Values are presented as mean \pm SD.

Abbreviation: BMI, body mass index. 
Table 2. Levels of various cytokines, MMPs, TIMPs, COX-2, and VEGF in endometriosis patients and controls

\begin{tabular}{lccr}
\hline Parameters & Endometriosis* & Controls* $^{*}$ & $P$ \\
\hline IL-1 $\beta$ (pg/mL) & $18.28 \pm 3.69$ & $3.88 \pm 1.91$ & $<0.001$ \\
TNF- $\alpha$ (pg/mL) & $117.43 \pm 20.73$ & $35.13 \pm 10.79$ & $<0.001$ \\
IFN- $\gamma(\mathrm{pg} / \mathrm{mL})$ & $16.35 \pm 3.95$ & $4.48 \pm 2.42$ & 0.012 \\
TGF- $\beta$ I (pg/mL) & $176.58 \pm 9.57$ & $110.84 \pm 6.64$ & $<0.001$ \\
IL-4 (pg/mL) & $178.40 \pm 14.70$ & $77.00 \pm 16.39$ & $<0.001$ \\
IL-10 (pg/mL) & $77.94 \pm 10.91$ & $18.73 \pm 2.67$ & $<0.001$ \\
IL-2 (pg/mL) & $46.52 \pm 4.42$ & $18.79 \pm 2.20$ & $<0.001$ \\
IL-6 (pg/mL) & $48.56 \pm 7.34$ & $15.61 \pm 4.33$ & $<0.001$ \\
IL-8 (pg/mL) & $97.61 \pm 5.25$ & $51.45 \pm 8.38$ & $<0.001$ \\
VEGF (ng/mL) & $546.93 \pm 23.99$ & $445.49 \pm 14.04$ & $<0.001$ \\
MMP-2 (ng/mL) & $128.24 \pm 8.20$ & $86.43 \pm 2.13$ & $<0.001$ \\
MMP-9 (ng/mL) & $154.12 \pm 8.75$ & $89.45 \pm 2.77$ & $<0.001$ \\
TIMP-1 (ng/mL) & $33.58 \pm 7.13$ & $128.51 \pm 46.60$ & $<0.001$ \\
TIMP-2 (ng/mL) & $30.71 \pm 3.09$ & $61.66 \pm 3.06$ & $<0.001$ \\
COX-2 (ng/mL) & $7.36 \pm 0.78$ & $1.21 \pm 0.22$ & $<0.001$ \\
\hline
\end{tabular}

*Values are presented as mean \pm SD.

Abbreviations: IL, interleukin; TNF, tumor necrosis factor; IFN, interferon; TGF, transforming growth factor; VEGF, vascular endothelial growth factor; MMP, matrix metalloproteinase; TIMP, tissue inhibitor of metalloproteinase; COX, cyclooxygenase.
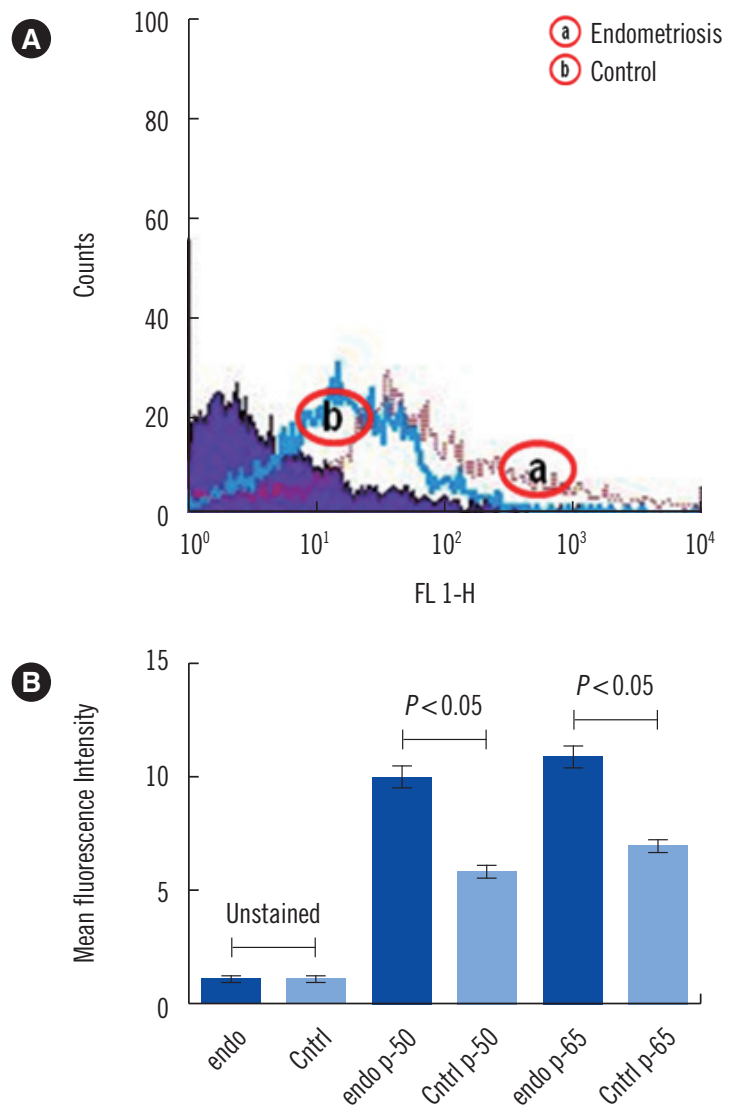

improved class separation (Fig. 2B). As the PLS-DA model has an inherent bias towards classification, the model was validated for its overfitting tendency using permutation test statistics (Fig. 2C). Our results indicate that the prediction capability of the original model is higher than that of the permutated models. The loading plot (Fig. 2D) and variable importance plot (VIP; Fig. 2E) generated from the PLS-DA analysis indicated that IL10 was the most important variable responsible for the classification of endometriosis cases.

\section{DISCUSSION}

Numerous studies have revealed disturbances in the immune response that are fundamental to the etiology and pathogenesis of endometriosis. The activation of NF- $\mathrm{kB}$ is principally regulated by ROS. Several rigorous studies have suggested the involvement of NF- $\mathrm{kB}$ transcriptional activity in endometriosis onset and progression [23, 24]. This is in agreement with our current findings that NF-kB expression is increased in patients with endometriosis than in controls (Fig. 1). NF-kB is also known to induce several cytokines $[25,26]$. It is evident that a selective block of $\mathrm{NF}-\mathrm{kB}$ activation results in restricted communication and secre-

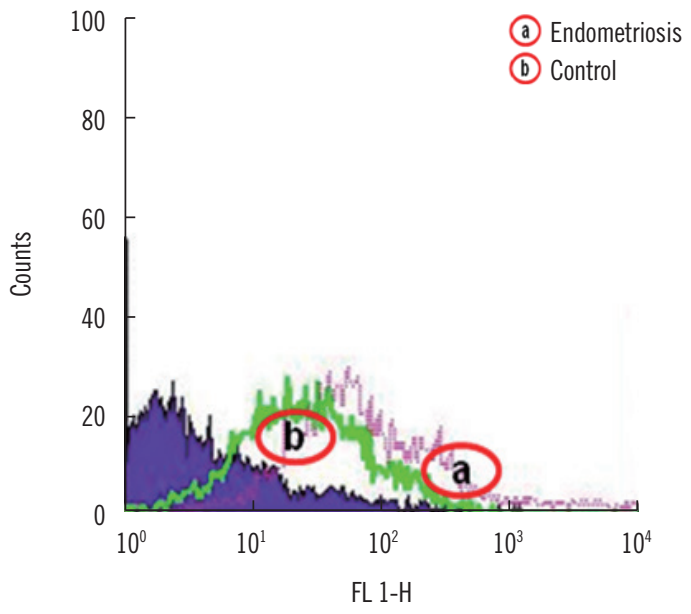

Fig. 1. Flow cytometry analysis of NF-kB activation. (A) Percentage of cells with NF-kB (p50 and p65) activation as measured by flow cytometry. (B) Graphical representation of cells with NF- $\mathrm{NB}$ activation as measured by flow cytometry. Abbreviations: Cntrl, control; Endo, endometriosis. 

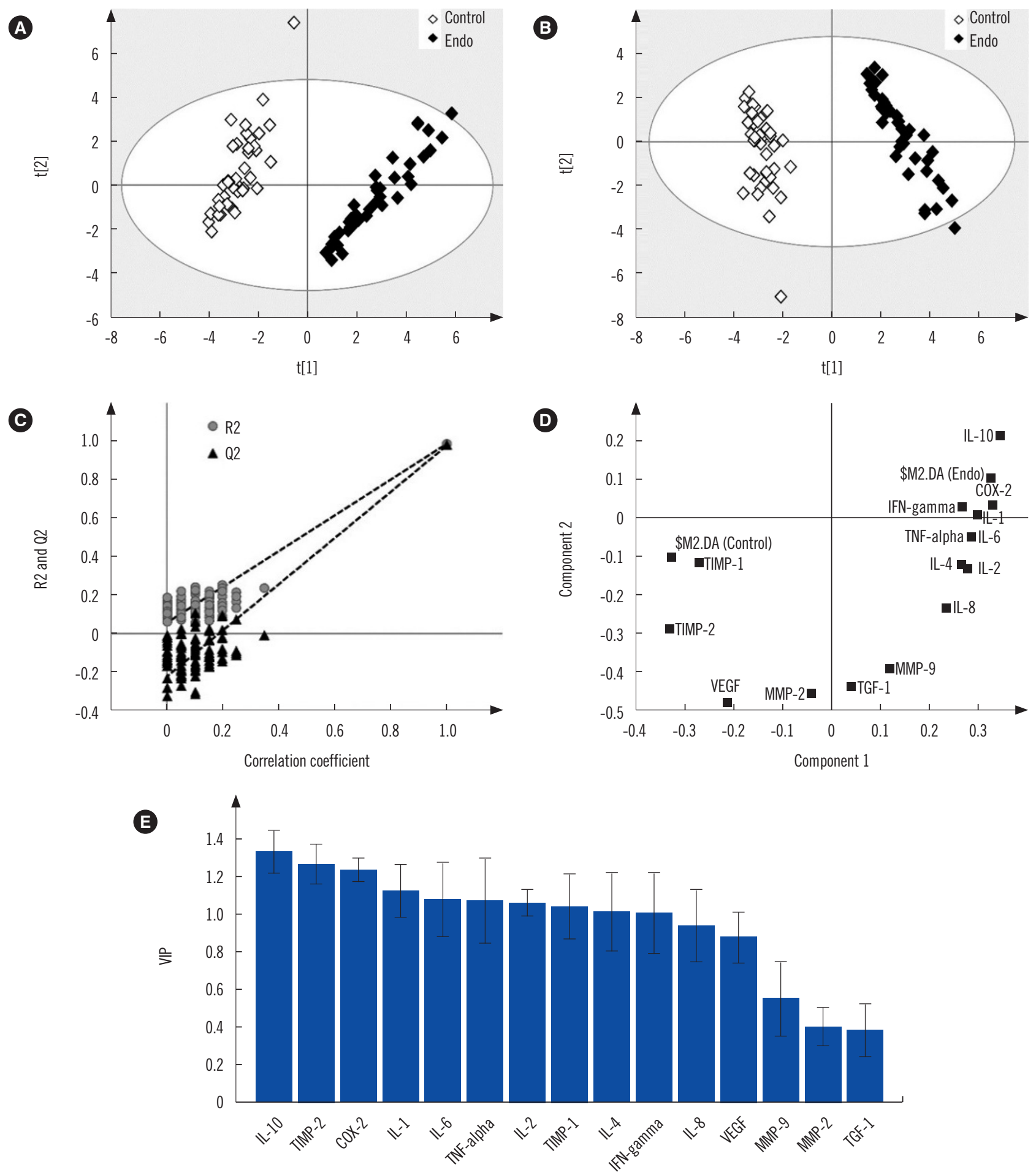

Var ID (Primary)

Fig. 2. Scores scatter plot of (A) PCA showing discrimination between endometriosis and controls, (B) PLS-DA showing improved discrimination between the two groups. (C) PLS-DA model cross-validation by permutation test statistics showing that the R2 (0.986) and Q2 (0.976) values of the original model are significantly greater than those of the 100 permutated models, indicating a good predictive ability. Variable extraction using (D) loading and (E) VIP plots showing that IL-10 is the most important variable for class separation.

Abbreviations: PCA, principal-component analysis; PLS-DA, partial least square discriminate analysis; VIP, variable importance plot; IL, interleukin; TNF, tumor necrosis factor; IFN, interferon; TGF, transforming growth factor; VEGF, vascular endothelial growth factor; MMP, matrix metalloproteinase; TIMP, tissue inhibitor of metalloproteinase; COX, cyclooxygenase. 
tion of several pro-inflammatory cytokines, TNF- $\alpha$, IL-6, and IL-8, which suggest the crucial role they play [27, 28]. Our results indicate a significant increase in the levels of pro-inflammatory cytokines, IL- $1 \beta$, TNF- $\alpha$, IFN- $\gamma$, and TGF- $\beta 1$ in patients with endometriosis (Table 2). This finding is also supported by several previous studies reporting higher levels of pro-inflammatory cytokines in patients with endometriosis [13, 14, 16]. However, a few studies have reported decreased expression of IFN- $\gamma$ [29, 30]. Increased levels of other cytokines, such as IL-2, IL-4, IL-6, IL8, and IL-10, was also observed in patients with endometriosis (Table 2). These findings are in agreement with previous studies demonstrating an increase in the expression of these cytokines in serum and peritoneal fluid from patients with endometriosis $[3,16,31]$.

Elevated expression of IL-10, a multifunctional cytokine, has been reported to play a major role in the establishment and maintenance of endometriosis [32]. This may be attributed to an increased level of pro-inflammatory cytokines such as IL-6 and TNF- $\alpha$, which are known to induce IL-10 [33]. Previous studies have also demonstrated the overexpression of COX-2 in endometriosis [34]. This enzyme is pivotal in endometriotic cell survival, migration, and invasion, and its expression has been shown to be induced by the presence of TGF- $\beta 1$ [35]. Our results indicated a significant increase in the expression of COX-2 in endometriosis. Furthermore, we also observed an increase in MMP-2 and -9 levels (Table 2), the activities of which are known to be regulated by COX-2 [36]. Moreover, decreased levels of TIMP-1 and -2 (Table 2) indicate an amplified MMP/TIMP ratio, which suggests an overall increase in ECM degradation in endometriotic women. Furthermore, the release of VEGF from the ECM is controlled by MMPs [37]; our results indicate that the elevated level of VEGF (Table 2) in patients with endometriosis promotes enhanced endometrial angiogenesis, which is reported to be an important factor for modulating endometriosis pathogenesis [38].

Multivariate statistical analysis was used to identify the most significant factor responsible for endometriosis pathogenesis. As evident from the PCA plot, the endometriosis and control groups are separated along with principal component 1 (t1) (Fig. 2A). An improved class separation was observed in the PLS-DA scores scatter plot (Fig. 2B). The loading plot (Fig. 2D) and VIP (Fig. 2E) generated from the PLS-DA analysis identified IL-10 as a significant variable capable of discriminating endometriosis samples from controls (Fig. 2C).

In summary, we investigated changes in different molecular events during endometriosis onset and progression. We hypothesize that excessive OS induces NF-kB, which stimulates cellu- lar processes leading to an increased level of cytokine production. In fact, NF-kB induces the Thelper (Th)1 and Th2 response immune system in cases of pelvic endometriosis [39]. The Th1 immune response stimulates pro-inflammatory cytokines, whereas the Th2 immune response induces anti-inflammatory cytokines. In particular, IL-10 expression is increased in an attempt to balance the Th1/Th2 response [40]. Thus, we hypothesize that an overexpressed pro-inflammatory and anti-inflammatory cytokine response may increase endometriosis progression. Furthermore, higher expression of COX-2 during endometriosis may be attributed to the overexpression of anti-inflammatory cytokines. COX2, in turn, up-regulates MMPs, leading to excessive ECM degradation. Additionally, reduced expression of TIMPs and imbalance between MMPs and TIMPs may increase ECM degradation. Excessive ECM degradation in conjunction with the impairment of cellular function may enhance the endometriotic lesion in an ectopic site for further disease progression. Although our results seem promising, elucidating the up- or down-regulation of these molecules in endometriosis is a complex issue and warrants further investigation with increased sample size. Further, a more localized investigation of these molecules in the peritoneal fluid and endometrial tissue is expected to provide a better understanding of the disease.

\section{ACKNOWLEDGEMENTS}

The authors are thankful to the staff of Pratiksha Hospital, Guwahati, Assam, India, for helping in the collection of samples.

\section{AUTHOR CONTRIBUTIONS}

AN, SKJ, and KC conceived and designed the experiments; AN performed the experiments; TK assisted with the experiments; PB and MD performed the multivariate statistical analysis; MD provided the multivariate statistical analysis tool; TW and PS assisted with patient recruitment and sample collection; AN and SKJ interpreted the data; SKJ provided reagents/materials/analysis tools; and AN, MD, and SKJ drafted the manuscript.

\section{CONFLICTS OF INTEREST}

None declared.

\section{RESEARCH FUNDING}

The study was funded by the Department of Biotechnology (DBT), 
Government of India, India (Research Project\# BT/PR16930/ NER/95/355).

\section{ORCID}

$\begin{array}{ll}\text { Amalesh Nanda } & \text { https://orcid.org/0000-0001-5406-2184 } \\ \text { Thangapandi K } & \text { https://orcid.org/0000-0001-5606-7819 } \\ \text { Priyanka Banerjee } & \text { https://orcid.org/0000-0001-6040-2675 } \\ \text { Mainak Dutta } & \text { https://orcid.org/0000-0003-0887-2893 } \\ \text { Tsering Wangdi } & \text { https://orcid.org/0000-0002-0271-3085 } \\ \text { Pramod Sharma } & \text { https://orcid.org/0000-0002-2599-4115 } \\ \text { Koel Chaudhury } & \text { https://orcid.org/0000-0002-9390-1179 } \\ \text { Saikat Kumar Jana } & \text { https://orcid.org/0000-0002-6294-925X }\end{array}$

\section{REFERENCES}

1. Giudice LC and Kao LC. Endometriosis. Lancet 2004;364:1789-99.

2. Li CY, Lang JH, Liu HY, Zhou HM. Expression of annexin-1 in patients with endometriosis. Chin Med J (Engl) 2008;121:927-31.

3. Gupta S, Agarwal A, Krajcir N, Alvarez JG. Role of oxidative stress in endometriosis. Reprod Biomed Online 2006;13:126-34.

4. Narayanan K, Balakrishnan A, Miyamoto S. NF-kappaB is essential for induction of pro-inflammatory cytokine genes by filarial parasitic sheath proteins. Mol Immunol 2000;37:115-23.

5. Harada T, Iwabe T, Terakawa N. Role of cytokines in endometriosis. Fertil Steril 2001;76:1-10.

6. Defrère S, González-Ramos R, Lousse JC, Colette S, Donnez O, Donnez $\mathrm{J}$, et al. Insights into iron and nuclear factor-kappa B (NF-kappaB) involvement in chronic inflammatory processes in peritoneal endometriosis. Histol Histopathol 2011;26:1083-92.

7. Banerjee P, Ghosh S, Dutta M, Subramani E, Khalpada J, Roychoudhury $S$, et al. Identification of key contributory factors responsible for vascular dysfunction in idiopathic recurrent spontaneous miscarriage. PLoS One 2013;8:e80940.

8. Krikun G. Endometriosis, angiogenesis and tissue factor. Scientifica (Cairo). 2012;2012:306830.

9. Eble JA, de Rezende FF. Redox-relevant aspects of the extracellular matrix and its cellular contacts via integrins. Antioxid Redox Signal 2014; 20:1977-93.

10. Fujisawa T, Hattori T, Takahashi K, Kuboki T, Yamashita A, Takigawa M. Cyclic mechanical stress induces extracellular matrix degradation in cultured chondrocytes via gene expression of matrix metalloproteinases and interleukin-1. J Biochem 1999;125:966-75.

11. Kang S, Cho S, Chung JH, Hammerberg C, Fisher GJ, Voorhees JJ. Inflammation and extracellular matrix degradation mediated by activated transcription factors nuclear factor-kappaB and activator protein-1 in inflammatory acne lesions in vivo. Am J Pathol 2005;166:1691-9.

12. Hoeben AN, Landuyt B, Highley MS, Wildiers H, Van Oosterom AT, De Bruijn EA. Vascular endothelial growth factor and angiogenesis. Pharmacol Rev 2004;56:549-80.

13. Collette T, Maheux R, Mailloux J, Akoum A. Increased expression of matrix metalloproteinase- 9 in the eutopic endometrial tissue of women with endometriosis. Hum Reprod 2006;21:3059-67.

14. Groothuis PG, Nap AW, Winterhager E, Grümmer R. Vascular development in endometriosis. Angiogenesis 2005;8:147-56.
15. Agarwal A, Aponte-Mellado A, Premkumar BJ, Shaman A, Gupta S. The effects of oxidative stress on female reproduction: a review. Reprod Biol Endocrinol 2012;10:49.

16. Gargett CE, Lederman F, Heryanto B, Gambino LS, Rogers PA. Focal vascular endothelial growth factor correlates with angiogenesis in human endometrium. Role of intravascular neutrophils. Hum Reprod 2001;16: 1065-75.

17. Ho HN, Wu MY, Chao KH, Chen CD, Chen SU, Chen HF, et al. Decrease in interferon gamma production and impairment of T-lymphocyte proliferation in peritoneal fluid of women with endometriosis. Am J Obstet Gynecol 1996;175:1236-41.

18. Szczepańska M, Koźlik J, Skrzypczak J, Mikołajczyk M. Oxidative stress may be a piece in the endometriosis puzzle. Fertil Steril 2003;79:128893.

19. Jana SK, Dutta M, Joshi M, Srivastava S, Chakravarty B, Chaudhury K. $1 \mathrm{H}$ NMR based targeted metabolite profiling for understanding the complex relationship connecting oxidative stress with endometriosis. Biomed Res Int 2013;2013:329058.

20. Dutta M, Singh B, Joshi M, Das D, Subramani E, Maan M, et al. Metabolomics reveals perturbations in endometrium and serum of minima and mild endometriosis. Sci Rep 2018;8:6466.

21. Nam JS, Cho MH, Lee GT, Park JS, Ahn CW, Cha BS, et al. The activation of NF-kappaB and AP-1 in peripheral blood mononuclear cells isolated from patients with diabetic nephropathy. Diabetes Res Clin Pract 2008;81:25-32.

22. Ichiyama T, Yoshitomi T, Nishikawa M, Fujiwara M, Matsubara T, Hayashi T, et al. NF-kappaB activation in peripheral blood monocytes/macrophages and T cells during acute Kawasaki disease. Clin Immunol 2001; 99:373-7.

23. Bedaiwy MA, Falcone T, Sharma RK, Goldberg JM, Attaran M, Nelson $\mathrm{DR}$, et al. Prediction of endometriosis with serum and peritoneal fluid markers: a prospective controlled trial. Hum Reprod 2002;17:426-31.

24. Wang D, Liu Y, Han J, Zai D, Ji M, Cheng W, et al. Puerarin suppresses invasion and vascularization of endometriosis tissue stimulated by $17 \beta$ estradiol. PLoS One 2011;6:e25011.

25. Cogswell JP, Godlevski MM, Wisely GB, Clay WC, Leesnitzer LM, Ways JP, et al. NF-kappa B regulates IL-1 beta transcription through a consensus NF-kappa B binding site and a nonconsensus CRE-like site. J Immunol 1994;153:712-23.

26. Sen R and Baltimore D. Multiple nuclear factors interact with the immunoglobulin enhancer sequences. Cell 1986;46:705-16.

27. Rhodus NL, Cheng B, Myers S, Miller L, Ho V, Ondrey F. The feasibility of monitoring NF-kappaB associated cytokines: TNF-alpha, IL-1alpha, IL-6, and IL-8 in whole saliva for the malignant transformation of oral lichen planus. Mol Carcinog 2005;44:77-82.

28. Tak PP and Firestein GS. NF-kappaB: a key role in inflammatory diseases. J Clin Invest 2001;107:7-11.

29. Barcz E, Kamiński P, Marianowski L. VEGF concentration in peritoneal fluid of patients with endometriosis. Ginekol Pol 2001;72:442-8.

30. Galleri L, Luisi S, Rotondi M, Romagnani P, Cobellis L, Serio M, et al. Low serum and peritoneal fluid concentration of interferon- $\gamma$-induced protein-10 (CXCL10) in women with endometriosis. Fertil Steril 2009; 91:331-4.

31. González-Ramos R, Defrère S, Devoto L. Nuclear factor-kappaB: a main regulator of inflammation and cell survival in endometriosis pathophysiology. Fertil Steril 2012;98:520-8

32. Suen JL, Chang Y, Chiu PR, Hsieh TH, Hsi E, Chen YC, et al. Serum level of IL-10 is increased in patients with endometriosis, and IL-10 promotes the growth of lesions in a murine model. Am J Pathol 2014;184: 464-71. 
Nanda A, et al.

Angiogenesis and ECM degradation in endometriosis

ANNALS OF

LABORATORY MEDICINE

33. Zhang JM and An J. Cytokines, inflammation, and pain. Int Anesthesiol Clin 2007;45:27-37.

34. González-Ramos R, Van Langendonckt A, Defrère S, Lousse JC, Colette $\mathrm{S}$, Devoto $\mathrm{L}$, et al. Involvement of the nuclear factor- $\mathrm{KB}$ pathway in the pathogenesis of endometriosis. Fertil Steril 2010;94:1985-94.

35. Ponce C, Torres M, Galleguillos C, Sovino H, Boric MA, Fuentes A, et al. Nuclear factor kappaB pathway and interleukin- 6 are affected in eutopic endometrium of women with endometriosis. Reproduction 2009;137: 727-37.

36. Galo S, Zúbor P, Szunyogh N, Kajo K, Macháleková K, Biringer K, et al. TNF-alpha serum levels in women with endometriosis: prospective clinical study. Ceska Gynekol 2005;70:286-90.
37. Mahecha AM and Wang $\mathrm{H}$. The influence of vascular endothelial growth factor-A and matrix metalloproteinase- 2 and -9 in angiogenesis, metastasis, and prognosis of endometrial cancer. Onco Targets Ther 2017;10: 4617-24.

38. Krikun G. Endometriosis, angiogenesis and tissue factor. Scientifica (Cairo) 2012;2012:306830.

39. Kobayashi H, Higashiura $Y$, Shigetomi $H$, Kajihara H. Pathogenesis of endometriosis: the role of initial infection and subsequent sterile inflammation (Review). Mol Med Rep 2014;9:9-15.

40. Fang D and Zhu J. Molecular switches for regulating the differentiation of inflammatory and IL-10-producing anti-inflammatory T-helper cells. Cell Mol Life Sci 2020;77:289-303. 